\title{
Planck Scale Cosmology and Asymptotic Safety in Resummed Quantum Gravity: An Estimate of $\Lambda$
}

\author{
B.F.L. Ward* \\ Dept. of Physics, \\ ${ }^{1}$ Baylor University, Waco, \\ ${ }^{2}$ TH Physics Unit, CERN, Geneva \\ 1 USA \\ ${ }^{2}$ Switzerland
}

\section{Introduction}

In Ref. [1], Weinberg suggested that the general theory of relativity may have a non-trivial UV fixed point, with a finite dimensional critical surface in the UV limit, so that it would be asymptotically safe with an S-matrix that depends on only a finite number of observable parameters. In Refs. [2-4], strong evidence has been calculated using Wilsonian [5-8] field-space exact renormalization group methods to support asymptotic safety for the Einstein-Hilbert theory. We have shown in Refs. [9-19] that the extension of the amplitude-based, exact resummation theory of Ref. [20] to the Einstein-Hilbert theory (we call the extension resummed quantum gravity) leads to UV fixed-point behavior for the dimensionless gravitational and cosmological constants, but with the bonus that the resummed theory is actually UV finite. More evidence for asymptotic safety for quantum gravity has been calculated using causal dynamical triangulated lattice methods in Ref. [21] . There is no known inconsistency between our analysis and Refs. [2-4,21]. Our results are also consistent with the results on leg renormalizability of quantum gravity in Refs. [23, 24].

The reader unfamiliar with the methods of Wilson in the context of the renormalization group may consult Refs. [2, 5-8] for the details of the approach. Here we stress that in the Wilsonian formulation of the renormalization group, it does not matter whether the theory under study is actually renormalizable because the idea is to thin the degrees of freedom to those relevant to the momentum scale $k$ under study. When one does this, the operators in the theory then fall into the classes of relevant, marginal and irrelevant operators as one studies the response of the theory to changes in the value of $k$. If the theory is renormalizable, then as $k \rightarrow \infty$ there will be a finite number of relevant or marginal operators in the effective action, yielding an S-matrix that depends on only a finite number of parameters. If the theory is non-renormalizable, there will be an infinite number of relevant or marginal operators in the effective action as $k \rightarrow \infty$. It was for this reason that the authors

\footnotetext{
${ }^{*}$ Work supported in part by NATO grant PST.CLG.980342.

${ }^{1}$ We also note that the model in Ref. [22] realizes many aspects of the effective field theory implied by the anomalous dimension of 2 at the UV-fixed point but it does so at the expense of violating Lorentz invariance.
} 
in Ref. [2-4] have chosen to use Wilsonian methods to study the Einstein-Hilbert theory, which is naively non-renormalizable by the standard power-counting arguments. What they find is that there are only a finite number of relevant or marginal operators in the effective action as $k \rightarrow \infty$, asymptotic safety. There is no contradiction with the naive expectation because the Wilsonian methods take into the account the non-perturbative changes in the scale dimensions of the theory's operators due to interactions. Unlike the methods in Refs. [2-4] which have unphysical cut-off dependence from thinning the degrees of freedom procedures and unphysical gauge dependence, our results have no such dependence on cut-offs or gauge choice. That we agree with the findings of Refs. [2-4] then strengthens these results. Contact with experiment is now in order.

Specifically, in Ref. [25], it has been argued that the approach in Refs. [2-4] to quantum gravity may provide a realization ${ }^{2}$ of the successful inflationary model [27, 28] of cosmology without the need of the inflaton scalar field: the attendant UV fixed point solution allows one to develop Planck scale cosmology that joins smoothly onto the standard Friedmann-Walker-Robertson classical descriptions so that one arrives at a quantum mechanical solution to the horizon, flatness, entropy and scale free spectrum problems. In Ref. [19], using the resummed quantum gravity theory [9-18], we recover the properties as used in Refs. [25] for the UV fixed point with "first principles" predictions for the fixed point values of the respective dimensionless gravitational and cosmological constants. Here, we carry the analysis one step further and arrive at a prediction for the observed cosmological constant $\Lambda$ in the context of the Planck scale cosmology of Refs. [25]. We comment on the reliability of the result as well, as it will be seen already to be relatively close to the observed value [29-31]. More such reflections, as they relate to an experimentally testable union of the original ideas of Bohr and Einstein, will be taken up elsewhere [32].

The discussion is organized as follows. In the next section we review the Planck scale cosmology presented in Refs. [25]. In Section 3 we review our results [19] for the dimensionless gravitational and cosmological constants at the UV fixed point. In Section 4, we combine the Planck scale cosmology scenario [25] with our results to predict the observed value of the cosmological constant $\Lambda$. Appendix 1 contains the evaluation of our gravitational resummation exponent.

\section{Planck scale cosmology}

More precisely, we recall the Einstein-Hilbert theory

$$
\mathcal{L}(x)=\frac{1}{2 \kappa^{2}} \sqrt{-g}(R-2 \Lambda)
$$

where $R$ is the curvature scalar, $g$ is the determinant of the metric of space-time $g_{\mu v}$, $\Lambda$ is the cosmological constant and $\kappa=\sqrt{8 \pi G_{N}}$ for Newton's constant $G_{N}$. Using the phenomenological exact renormalization group for the Wilsonian [5-8] coarse grained effective average action in field space, the authors in Ref. [25] have argued that the attendant running Newton constant $G_{N}(k)$ and running cosmological constant $\Lambda(k)$ approach $U V$ fixed points as $k$ goes to infinity in the deep Euclidean regime:

$$
k^{2} G_{N}(k) \rightarrow g_{*}, \Lambda(k) \rightarrow \lambda_{*} k^{2}
$$

\footnotetext{
$\overline{2}$ The attendant scale choice $k \sim 1 / t$ used in Refs. [25] was also proposed in Ref. [26].
} 
for $k \rightarrow \infty$.

The contact with cosmology then proceeds as follows. Using a phenomenological connection between the momentum scale $k$ characterizing the coarseness of the Wilsonian graininess of the average effective action and the cosmological time $t, k(t)=\frac{\xi}{t}$ for $\xi>0$, the authors in Refs. [25] show that the standard cosmological equations admit of the following extension:

$$
\begin{aligned}
\left(\frac{\dot{a}}{a}\right)^{2}+\frac{K}{a^{2}} & =\frac{1}{3} \Lambda+\frac{8 \pi}{3} G_{N} \rho \\
\dot{\rho}+3(1+\omega) \frac{\dot{a}}{a} \rho & =0 \\
\dot{\Lambda}+8 \pi \rho G_{N} & =0 \\
G_{N}(t) & =G_{N}(k(t)) \\
\Lambda(t) & =\Lambda(k(t))
\end{aligned}
$$

for the density $\rho$ and scale factor $a(t)$ with the Robertson-Walker metric representation as

$$
d s^{2}=d t^{2}-a(t)^{2}\left(\frac{d r^{2}}{1-K r^{2}}+r^{2}\left(d \theta^{2}+\sin ^{2} \theta d \phi^{2}\right)\right)
$$

so that $K=0,1,-1$ correspond respectively to flat, spherical and pseudo-spherical 3-spaces for constant time $t$. The equation of state is

$$
p(t)=\omega \rho(t)
$$

where $p$ is the pressure.

Using the UV fixed points for $g_{*}$ and $\lambda_{*}$, the authors in Refs. [25] show that the extended cosmological system given above admits, for $K=0$, a solution in the Planck regime where $0 \leq$ $t \leq t_{\text {class }}$, with $t_{\text {class }}$ a "few" times the Planck time $t_{P l}$, which joins smoothly onto a solution in the classical regime, $t>t_{\text {class }}$, which coincides with standard Friedmann-Robertson-Walker phenomenology but with the horizon, flatness, scale free Harrison-Zeldovich spectrum, and entropy problems all solved purely by Planck scale quantum physics. We now review the results in Refs. [19] for these UV limits as implied by resummed quantum gravity theory as presented in [9-18] and show how to use them to predict the current value of $\Lambda$. In this way, we put the arguments in Refs. [25] on a more rigorous theoretical basis.

\section{3. $g_{*}$ and $\lambda_{*}$ in resummed quantum gravity}

We start with the prediction for $g_{*}$, which we already presented in Refs. [9-19]. For the sake of completeness, let us we recapitulate the main steps in the calculation. Referring to Fig. 1, we have shown in Refs. [9-18] that the large virtual IR effects in the respective loop integrals for the scalar propagator in quantum general relativity can be resummed to the exact result

$$
\left.i \Delta_{F}^{\prime}(k)\right|_{\text {resummed }}=\frac{i e^{B_{g}^{\prime \prime}(k)}}{\left(k^{2}-m^{2}-\Sigma_{s}^{\prime}+i \epsilon\right)}
$$




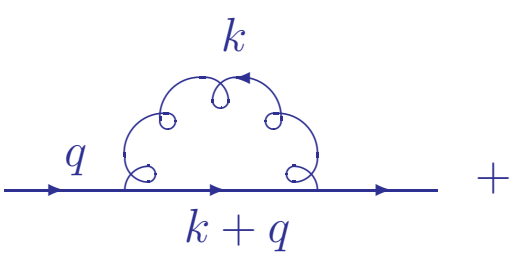

(a)

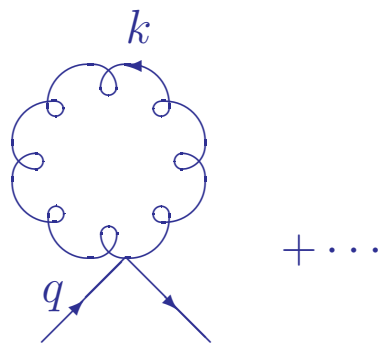

(b)

Fig. 1. Graviton loop contributions to the scalar propagator. $q$ is the 4-momentum of the scalar.

for $\left(\Delta=k^{2}-m^{2}\right)$

$$
\begin{aligned}
B_{g}^{\prime \prime}(k)= & -2 i \kappa^{2} k^{4} \frac{\int d^{4} \ell}{16 \pi^{4}} \frac{1}{\ell^{2}-\lambda^{2}+i \epsilon} \\
& \frac{1}{\left(\ell^{2}+2 \ell k+\Delta+i \epsilon\right)^{2}} \\
= & \frac{\kappa^{2}\left|k^{2}\right|}{8 \pi^{2}} \ln \left(\frac{m^{2}}{m^{2}+\left|k^{2}\right|}\right),
\end{aligned}
$$

where the latter form holds for the UV regime, so that (5) the resummed scalar propagator falls faster than any power of $\left|k^{2}\right|$. An analogous result [9-18] holds for $m=0$ (See Appendix 1.). As $\Sigma_{s}^{\prime}$, the residual self-energy function, starts in $\mathcal{O}\left(\kappa^{2}\right)$, we may drop it in calculating one-loop effects. It follows that, when the respective analogs of $\left.i \Delta_{F}^{\prime}(k)\right|_{\text {resummed }}$ are used for the elementary particles, all quantum gravity loop corrections are UV finite [9-18].

We stress that our resummed scalar propagator representation (5) is not limited to the regime where $k^{2} \cong m^{2}$ but is an identity that holds for all $k^{2}$ - see Refs. [9-18]. This is readily shown as follows. If we invert both sides of (5) and recall the formula for the exact inverse propagator, we get

$$
\Delta_{F}^{-1}(k)-\Sigma_{s}(k)=\left(\Delta_{F}^{-1}(k)-\Sigma_{S}^{\prime}(k)\right) e^{-B_{g}^{\prime \prime}(k)}
$$

where the free inverse propagator is $\Delta_{F}^{-1}(k)=\Delta(k)+i \epsilon$ and $\Sigma_{s}(k)$ is the exact proper self-energy part. We introduce here the loop expansions

$$
\begin{aligned}
& \Sigma_{s}(k)=\sum_{n=1}^{\infty} \Sigma_{s, n}(k)\left(\frac{\kappa^{2}}{4 \pi^{2}}\right)^{n} \\
& \Sigma_{s}^{\prime}(k)=\sum_{n=1}^{\infty} \Sigma_{s, n}^{\prime}(k)\left(\frac{\kappa^{2}}{4 \pi^{2}}\right)^{n}
\end{aligned}
$$

and we get, from elementary algebra, the exact relation

$$
-\Sigma_{s, n}(k)=-\sum_{j=0}^{n} \Sigma_{s, j}^{\prime}(k)\left(\frac{-4 \pi^{2} B_{g}^{\prime \prime}(k)}{\kappa^{2}}\right)^{n-j} /(n-j) !
$$


where we define for convenience $-\Sigma_{s, 0}(k)=-\Sigma_{s, 0}^{\prime}(k)=\Delta_{F}^{-1}(k)$. This proves that every Feynman diagram contribution to $\Sigma_{s}(k)$ corresponds to a unique contribution to $\Sigma_{s}^{\prime}(k)$ to all orders in $\kappa^{2} /(4 \pi)$ for all values of $k^{2}$. QED.

When we use our resummed propagator results, as extended to all the particles in the SM Lagrangian and to the graviton itself, , working now with the complete theory (see Refs. [919]) of (1) plus the SM Lagrangian written in diffeomorphism invariant form as explained in Refs. [9-18],

$$
\mathcal{L}(x)=\frac{1}{2 \kappa^{2}} \sqrt{-g}(R-2 \Lambda)+\sqrt{-g} L_{S M}^{\mathcal{G}}(x)
$$

where $L_{S M}^{\mathcal{G}}(x)$ is the SM Lagrangian written in diffeomorphism invariant form as explained in Refs. [9-18], the denominator of the graviton propagator becomes [9-18] $\left(M_{P l}\right.$ is the Planck mass)

$$
q^{2}+\Sigma^{T}\left(q^{2}\right)+i \epsilon \cong q^{2}-q^{4} \frac{c_{2, e f f}}{360 \pi M_{P l}^{2}}
$$

for

$$
\begin{aligned}
c_{2, e f f} & =\sum_{\text {SM particles j }} n_{j} I_{2}\left(\lambda_{c}(j)\right) \\
& \cong 2.56 \times 10^{4}
\end{aligned}
$$

with $I_{2}$ given in Refs. [9-18] by

$$
I_{2}\left(\lambda_{c}\right)=\int_{0}^{\infty} d x x^{3}(1+x)^{-4-\lambda_{c} x}
$$

and with $\lambda_{c}(j)=\frac{2 m_{j}^{2}}{\pi M_{P l}^{2}} \cdot n_{j}$ is the number of effective degrees of freedom [9-18] of particle $j$ of mass $m_{j}$. In $c_{2, \text { eff }}$ in (11), we take the SM masses as explained in Refs. [9-19] following Refs. [29-31, 33-35] : for the now presumed three massive neutrinos [33], we estimate a mass at $\sim 3 \mathrm{eV}$; for the remaining members of the known three generations of Dirac fermions $\{e, \mu, \tau, u, d, s, c, b, t\}$, we use [34] $m_{e} \cong 0.51 \mathrm{MeV}, m_{\mu} \cong 0.106 \mathrm{GeV}, m_{\tau} \cong 1.78 \mathrm{GeV}, m_{u} \cong 5.1$ $\mathrm{MeV}, m_{d} \cong 8.9 \mathrm{MeV}, m_{s} \cong 0.17 \mathrm{GeV}, m_{c} \cong 1.3 \mathrm{GeV}, m_{b} \cong 4.5 \mathrm{GeV}$ and $m_{t} \cong 174 \mathrm{GeV}$ and for the massive vector bosons $W^{ \pm}, Z$ we use the masses $M_{W} \cong 80.4 \mathrm{GeV}, M_{Z} \cong 91.19 \mathrm{GeV}$, respectively. We set the Higgs mass at $m_{H} \cong 120 \mathrm{GeV}$, in view of the limit from LEP2 [35]. We note that (see the Appendix 1 here and the Appendix 1 in Ref. [9]) when the rest mass of particle $j$ is zero, such as it is for the photon and the gluon, the value of $m_{j}$ turns-out to be $\sqrt{2}$ times the gravitational infrared cut-off mass [29-31], which is $m_{g} \cong 3.1 \times 10^{-33} \mathrm{eV}$. We also note that from Ref.[36] it also follows that the value of $n_{j}$ for the graviton and its attendant ghost is 42 . For $\lambda_{c} \rightarrow 0$, we have found the approximate representation

$$
I_{2}\left(\lambda_{c}\right) \cong \ln \frac{1}{\lambda_{c}}-\ln \ln \frac{1}{\lambda_{c}}-\frac{\ln \ln \frac{1}{\lambda_{c}}}{\ln \frac{1}{\lambda_{c}}-\ln \ln \frac{1}{\lambda_{c}}}-\frac{11}{6} .
$$

We thus identify (we use $G_{N}$ for $G_{N}(0)$ )

$$
G_{N}(k)=G_{N} /\left(1+\frac{c_{2, e f f} k^{2}}{360 \pi M_{P l}^{2}}\right)
$$


and compute the UV limit $g_{*}$ as

$$
g_{*}=\lim _{k^{2} \rightarrow \infty} k^{2} G_{N}\left(k^{2}\right)=\frac{360 \pi}{c_{2, e f f}} \cong 0.0442 .
$$

We stress that this result has no threshold/cut-off or gauge effects in it. a pure property of the known world.

Turning now to $\lambda_{*}$, we use Einstein's equation

$$
G_{\mu v}+\Lambda g_{\mu v}=-\kappa^{2} T_{\mu v}
$$

in a standard notation where $G_{\mu \nu}=R_{\mu v}-\frac{1}{2} R g_{\mu v}, R_{\mu v}$ is the contracted Riemann tensor, and $T_{\mu \nu}$ is the energy-momentum tensor. Working with the representation $g_{\mu v}=\eta_{\mu \nu}+2 \kappa h_{\mu \nu}$ for the flat Minkowski metric $\eta_{\mu v}=\operatorname{diag}(1,-1,-1,-1)$ we may isolate $\Lambda$ in Einstein's equation by evaluating its VEV (vacuum expectation value). For any bosonic quantum field $\varphi$ we use the point-splitting definition (here, : : denotes normal ordering)

$$
\begin{aligned}
\varphi(0) \varphi(0) & =\lim _{\epsilon \rightarrow 0} \varphi(\epsilon) \varphi(0) \\
& =\lim _{\epsilon \rightarrow 0} T(\varphi(\epsilon) \varphi(0)) \\
& =\lim _{\epsilon \rightarrow 0}\{:(\varphi(\epsilon) \varphi(0)):+<0|T(\varphi(\epsilon) \varphi(0))| 0>\}
\end{aligned}
$$

where the limit is taken with time-like $\epsilon \equiv(\epsilon, \overrightarrow{0}) \rightarrow(0,0,0,0) \equiv 0$ respectively. A scalar then makes the contribution [9-18] to $\Lambda$ given by ${ }^{3}$

$$
\begin{aligned}
\Lambda_{s} & =-8 \pi G_{N} \frac{\int d^{4} k}{2(2 \pi)^{4}} \frac{\left(2 k_{0}^{2}\right) e^{-\lambda_{c}\left(k^{2} /\left(2 m^{2}\right)\right) \ln \left(k^{2} / m^{2}+1\right)}}{k^{2}+m^{2}} \\
& \cong-8 \pi G_{N}\left[\frac{1}{G_{N}^{2} 64 \rho^{2}}\right],
\end{aligned}
$$

where $\rho=\ln \frac{2}{\lambda_{c}}$ and we have used the calculus of Refs. [9-18]. We note that the standard equal-time (anti-)commutation relations algebra realizations then show that a Dirac fermion contributes -4 times $\Lambda_{s}$ to $\Lambda$. The deep UV limit of $\Lambda$ then becomes

$$
\begin{aligned}
\Lambda(k) & \underset{k^{2} \rightarrow \infty}{\longrightarrow} k^{2} \lambda_{* \prime} \\
\lambda_{*} & =-\frac{c_{2, e f f}}{2880} \sum_{j}(-1)^{F_{j}} n_{j} / \rho_{j}^{2} \\
& \cong 0.0817
\end{aligned}
$$

where $F_{j}$ is the fermion number of $j$ and $\rho_{j}=\rho\left(\lambda_{c}\left(m_{j}\right)\right)$. We see again that $\lambda_{*}$ is free of threshold/cut-off effects and of gauge artifacts and is a pure prediction of our known

\footnotetext{
${ }^{3}$ We note the use here in the integrand of $2 k_{0}^{2}$ rather than the $2\left(\vec{k}^{2}+m^{2}\right)$ in Ref. [19], to be consistent with $\omega=-1$ [37] for the vacuum stress-energy tensor.
} 
world $-\lambda_{*}$ would vanish in an exactly supersymmetric theory. Our results for $\left(g_{*}, \lambda_{*}\right)$ agree qualitatively with those in Refs. [2, 25].

For reference, we note that, if we restrict our resummed quantum gravity calculations above for $g_{*}, \lambda_{*}$ to the pure gravity theory with no SM matter fields, we get the results

$$
g_{*}=.0533, \lambda_{*}=-.000189
$$

. We see that our results suggest that there is still significant cut-off effects in the results used for $g_{*}, \lambda_{*}$ in Refs. [2, 25], which already seem to include an effective matter contribution when viewed from our resummed quantum gravity perspective, as an artifact of the obvious gauge and cut-off dependences of the results. Indeed, from a purely quantum field theoretic point of view, the cut-off action is

$$
\Delta_{k} S(h, C, \bar{C} ; \bar{g})=\frac{1}{2}<h, \mathcal{R}_{k}^{\text {grav }} h>+<\bar{C}, \mathcal{R}_{k}^{\text {gh }} C>
$$

where $\bar{g}$ is the general background metric, which is the Minkowski space metric $\eta$ here, and $C, \bar{C}$ are the ghost fields and the operators $\mathcal{R}_{k}^{\text {grav }}, \mathcal{R}_{k}^{\text {gh }}$ implement the course graining as they satisfy the limits

$$
\begin{gathered}
\lim _{p^{2} / k^{2} \rightarrow \infty} \mathcal{R}_{k}=0, \\
\lim _{p^{2} / k^{2} \rightarrow 0} \mathcal{R}_{k} \rightarrow \mathfrak{Z}_{k} k^{2},
\end{gathered}
$$

for some $\mathfrak{Z}_{k}$ [2]. Here, the inner product is that defined in the second paper in Refs. [2] in its Eqs. $(2.14,2.15,2.19)$. The result is that the modes with $p \lesssim k$ have a shift of their vacuum energy by the cut-off operator. There is therefore no disagreement in principle between our gauge invariant and cut-off independent results and the gauge dependent and cut-off dependent results in Refs. [2, 25].

\section{An estimate of $\Lambda$}

To estimate the value of $\Lambda$ today, we take the normal-ordered form of Einstein's equation,

$$
: G_{\mu \nu}:+\Lambda: g_{\mu \nu}:=-\kappa^{2}: T_{\mu \nu}:
$$

The coherent state representation of the thermal density matrix then gives the Einstein equation in the form of thermally averaged quantities with $\Lambda$ given by our result above in lowest order. Taking the transition time between the Planck regime and the classical Friedmann-Robertson-Walker regime at $t_{t r} \sim 25 t_{P l}$ from Refs. [25], we introduce

$$
\begin{aligned}
\rho_{\Lambda}\left(t_{t r}\right) & \equiv \frac{\Lambda\left(t_{t r}\right)}{8 \pi G_{N}\left(t_{t r}\right)} \\
& =\frac{-M_{P l}^{4}\left(k_{t r}\right)}{64} \sum_{j} \frac{(-1)^{F} n_{j}}{\rho_{j}^{2}}
\end{aligned}
$$


and use the arguments in Refs. [38] ( $t_{e q}$ is the time of matter-radiation equality) to get the first principles estimate, from the method of the operator field,

$$
\begin{aligned}
& \rho_{\Lambda}\left(t_{0}\right) \cong \frac{-M_{P l}^{4}\left(1+c_{2, e f f} k_{t r}^{2} /\left(360 \pi M_{P l}^{2}\right)\right)^{2}}{64} \sum_{j} \frac{(-1)^{F} n_{j}}{\rho_{j}^{2}} \\
& \times\left[\frac{t_{t r}^{2}}{t_{e q}^{2}} \times\left(\frac{t_{e q}^{2 / 3}}{t_{0}^{2 / 3}}\right)^{3}\right] \\
& \cong \frac{-M_{P l}^{2}(1.0362)^{2}\left(-9.197 \times 10^{-3}\right)}{64} \frac{(25)^{2}}{t_{0}^{2}} \\
& \cong\left(2.400 \times 10^{-3} e V\right)^{4} .
\end{aligned}
$$

where we take the age of the universe to be $t_{0} \cong 13.7 \times 10^{9} \mathrm{yrs}$. In the latter estimate, the first factor in the square bracket comes from the period from $t_{t r}$ to $t_{e q}$ (radiation dominated) and the second factor comes from the period from $t_{e q}$ to $t_{0}$ (matter dominated) ${ }^{4}$. This estimate should be compared with the experimental result $\left.[29-31]^{5} \rho_{\Lambda}\left(t_{0}\right)\right|_{\text {expt }} \cong\left(2.368 \times 10^{-3} \mathrm{eV}(1 \pm\right.$ $0.023))^{4}$.

To sum up, our estimate, while it is definitely encouraging, is not a precision prediction, as possible hitherto unseen degrees of freedom have not been included and $t_{t r}$ is not precise, yet.

\section{Acknowledgments}

We thank Profs. L. Alvarez-Gaume and W. Hollik for the support and kind hospitality of the CERN TH Division and the Werner-Heisenberg-Institut, MPI, Munich, respectively, where a part of this work was done.

\section{Appendix: Gravitational infrared exponent}

In the text, we use several limits of the gravitational infrared exponent $B_{g}^{\prime \prime}$ defined in (6). This appendix contains these evaluations for completeness.

We have to consider

$$
-B_{g}^{\prime \prime}(p)=\frac{2 i \kappa^{2} p^{4}}{16 \pi^{4}} \int \frac{d^{4} k}{\left(k^{2}-\lambda^{2}+i \epsilon\right)} \frac{1}{\left(k^{2}-2 k p+\Delta+i \epsilon\right)^{2}}
$$

where $\Delta=p^{2}-m^{2}$. The integral on the RHS of (24) is given by

$$
\begin{aligned}
I & =\int \frac{d^{4} k}{\left(k^{2}-\lambda^{2}+i \epsilon\right)} \frac{1}{\left(k^{2}-2 k p+\Delta+i \epsilon\right)^{2}} \\
& =\frac{-i \pi^{2}}{p^{2}} \frac{1}{x_{+}-x_{-}}\left[x_{+} \ln \left(1-1 /\left(\sqrt{2} x_{+}\right)\right)-x_{-} \ln \left(1-1 /\left(\sqrt{2} x_{-}\right)\right)\right]
\end{aligned}
$$

\footnotetext{
${ }^{4}$ The method of the operator field forces the vacuum energies to follow the same scaling as the non-vacuum excitations.

${ }^{5}$ See also Ref. [39] for an analysis that suggests a value for $\rho_{\Lambda}\left(t_{0}\right)$ that is qualitatively similar to this experimental result.
} 
with

$$
x_{ \pm}=\frac{1}{2 \sqrt{2}}\left(\bar{\Delta}+\bar{\lambda}^{2} \pm\left(\left(\bar{\Delta}+\bar{\lambda}^{2}\right)^{2}-4\left(\bar{\lambda}^{2}-i \bar{\epsilon}\right)\right)^{1 / 2}\right)
$$

for $\bar{\Delta}=1-m^{2} / p^{2}, \bar{\lambda}^{2}=\lambda^{2} / p^{2}$ and $\bar{\epsilon}=\epsilon / p^{2}$. In this way, we arrive at the results, for $p^{2}<0$,

$$
B_{g}^{\prime \prime}(p)= \begin{cases}\frac{\kappa^{2}\left|p^{2}\right|}{8 \pi^{2}} \ln \left(\frac{m^{2}}{m^{2}+\left|p^{2}\right|}\right), & m \neq 0 \\ \frac{\kappa^{2}\left|p^{2}\right|}{8 \pi^{2}} \ln \left(\frac{m_{g}^{2}}{m_{g}^{2}\left|p^{2}\right|}\right), & m=m_{g}=\lambda \\ \frac{2 \kappa^{2}\left|p^{2}\right|}{8 \pi^{2}} \ln \left(\frac{m_{g}^{2}}{\left|p^{2}\right|}\right), m=0, m_{g}=\lambda\end{cases}
$$

where we have made more explicit the presence of the observed small mass, $m_{g}$, of the graviton. When $m=0$ and one wants to use dimensional regularization for the IR regime instead of $m_{g}$, we normalize the propagator at a Euclidean point $k^{2}=-\mu^{2}$ and use standard factorization arguments [40-44] to take the factorized result for $B_{g}^{\prime \prime}$ from (26) as

$$
\left.B_{g}^{\prime \prime}(p)\right|_{\text {factorized }}=\frac{2 \kappa^{2}\left|p^{2}\right|}{8 \pi^{2}} \ln \left(\frac{\left|\mu^{2}\right|}{\left|p^{2}\right|}\right), m=0, m_{g}=0 .
$$

In physical applications, such mass singularities are absorbed by the definition of the initial state "parton" densities and/or are canceled by the KLN theorem in the final state; we do not exponentiate them in the exactly massless case.

We stress that the standard analytic properties of the 1PI 2pt functions obtain here, as we use standard Feynman rules. Wick rotation changes the Minkowski space Feynman loop integral $\int d^{4} k$ with $k=\left(k^{0}, k^{1}, k^{2}, k^{3}\right)$ for real $k^{j}$ and $k^{2}=k^{0^{2}}-k^{1^{2}}-k^{2}-k^{3^{2}}$ into the integral $i \int d^{4} k_{E}$ with $k=\left(i k^{0}, k^{1}, k^{2}, k^{3}\right)$ and $k^{2}=-k^{0^{2}}-k^{1^{2}}-k^{2^{2}}-k^{3^{2}} \equiv-k_{E}^{2}$ with $k_{E}$ the Euclidean 4 -vector $k_{E}=\left(k^{0}, k^{1}, k^{2}, k^{3}\right)$ with metric $\delta_{\mu v}=\operatorname{diag}(1,1,1,1)$. Thus our results rigorously correspond to $\left|p^{2}\right|=-p^{2}$ in (26), (27) with $m^{2}$ replaced with $m^{2}-i \epsilon$, with $\epsilon \downarrow 0$, following Feynman, for $p^{2}<0$; by Wick rotation this is the regime relevant to the UV behavior of the Feynman loop integral. Standard complex variables theory then uniquely specifies our exponent for any value of $p^{2}$.

\section{References}

[1] S. Weinberg, in General Relativity, an Einstein Centenary Survey, eds. S. W. Hawking and W. Israel, (Cambridge Univ. Press, Cambridge, 1979).

[2] M. Reuter, Phys. Rev. D 57 (1998) 971; O. Lauscher and M. Reuter, ibid. 66 (2002) 025026; A. Bonanno and M. Reuter, ibid. 62 (2000) 043008, and references therein.

[3] D. F. Litim, Phys. Rev. Lett. 92(2004) 201301; Phys. Rev. D 64 (2001) 105007, and references therein.

[4] R. Percacci and D. Perini, Phys. Rev. D 68 (2003) 044018.

[5] K. G. Wilson, Phys. Rev. B 4 (1971) 3174, 3184; K. G. Wilson, J.Kogut, Phys. Rep. 12 (1974) 75.

[6] F. Wegner and A. Houghton, Phys. Rev. A 8(1973) 401.

[7] S. Weinberg, "Critical Phenomena for Field Theorists", Erice Subnucl. Phys. (1976) 1.

[8] J. Polchinski, Nucl. Phys. B 231 (1984) 269.

[9] B.F.L. Ward, Open Nucl.Part.Phys.Jour. 2(2009) 1. 
[10] B.F.L. Ward, Mod. Phys. Lett. A 17 (2002) 237.

[11] B.F.L. Ward, Mod. Phys. Lett. A 19 (2004) 14.

[12] B.F.L. Ward, J. Cos. Astropart. Phys. 0402 (2004) 011.

[13] B.F.L. Ward, Acta Phys. Polon. B37 (2006) 1967.

[14] B.F.L. Ward, Acta Phys. Polon. B37 (2006) 347.

[15] B.F.L. Ward, hep-ph/0502104; in Focus on Black Hole Research, ed. P.V. Kreitler,(Nova Sci. Publ., Inc., New York, 2006) p. 95.

[16] B.F.L. Ward, hep-ph/0411050; Int. J. Mod. Phys. A20 (2005) 3502.

[17] B.F.L. Ward, hep-ph/0411049; Int. J. Mod. Phys. A20 (2005) 3128.

[18] B.F.L. Ward, hep-ph/0410273; in Proc. ICHEP 2004, vol. 1, eds. H. Chen et al.,(World Sci. Publ. Co., Singapore, 2005) p. 419 and references therein.

[19] B.F.L. Ward, Mod. Phys. Lett. A 23 (2008) 3299.

[20] D. R. Yennie, S. C. Frautschi, and H. Suura, Ann. Phys. 13 (1961) 379; see also K. T. Mahanthappa, Phys. Rev. 126 (1962) 329, for a related analysis.

[21] J. Ambjorn et al., Phys. Lett. B 690 (2010) 420, and references therein.

[22] P. Horava, Phys. Rev. D 79 (2009) 084008.

[23] D. Kreimer, Ann. Phys. 321 (2006) 2757.

[24] D. Kreimer, Ann. Phys. 323 (2008) 49.

[25] A. Bonanno and M. Reuter, Phys. Rev. D 65 (2002) 043508; Jour. Phys. Conf. Ser. 140 (2008) 012008, and references therein.

[26] I.L. Shapiro and J. Sola, Phys. Lett. B 475 (2000) 236.

[27] See for example A. H. Guth and D.I. Kaiser, Science 307 (2005) 884; A. H. Guth, Phys. Rev. D 23 (1981) 347, and references therein.

[28] See for example A. Linde, Lect. Notes. Phys. 738 (2008) 1, and references therein.

[29] A.G. Riess et al., Astron. Jour. 116 (1998) 1009.

[30] S. Perlmutter et al., Astrophys. J. 517 (1999) 565.

[31] C. Amsler et al., Phys. Lett. B 667 (2008) 1 and, references therein.

[32] B.F.L. Ward, to appear.

[33] See for example D. Wark, in Proc. ICHEP02, eds. S. Bentvelsen et al., (North-Holland,Amsterdam, 2003), Nucl. Phys. B (Proc. Suppl.) 117 (2003) 164; M. C. Gonzalez-Garcia, hep-ph/0211054, in Proc. ICHEP02, eds. S. Bentvelsen et al., (North-Holland,Amsterdam, 2003), Nucl. Phys. B (Proc. Suppl.) 117 (2003) 186, and references therein.

[34] K. Hagiwara et al., Phys. Rev. D 66 (2002) 010001; S. Eidelman et al., Phys. Lett. B 592 (2004) 1; H. Leutwyler and J. Gasser, Phys. Rept. 87 (1982) 77, and references therein.

[35] D. Abbaneo et al., hep-ex/0212036; M. Gruenewald, hep-ex/0210003, in Proc. ICHEP02, eds. S. Bentvelsen et al., (North-Holland,Amsterdam, 2003), Nucl. Phys. B Proc. Suppl. 117(2003) 280.

[36] G. 't Hooft and M. Veltman, Ann. Inst. Henri Poincare XX (1974) 69.

[37] Ya. B. Zeldovich, Sov. Phys. Uspekhi 11 (1968) 381.

[38] V. Branchina and D. Zappala, G. R. Gravit. 42 (2010) 141; arXiv:1005.3657, and references therein.

[39] J. Sola, J. Phys. A 41 (2008) 164066.

[40] R.K. Ellis et al., Phys. Lett. B 78 (1978) 281-4.

[41] R.K. Ellis et al., Nucl. Phys. B 152 (1979) 285-329.

[42] D. Amati, R. Petronzio and G. Veneziano G, Nucl. Phys. Bbf 146 (1978) 29-49.

[43] S. Libby and G. Sterman G, Phys. Rev. D 18 (1978) 3252-68.

[44] A. Mueller, Phys. Rev. D 18 (1978) 3705-27. 


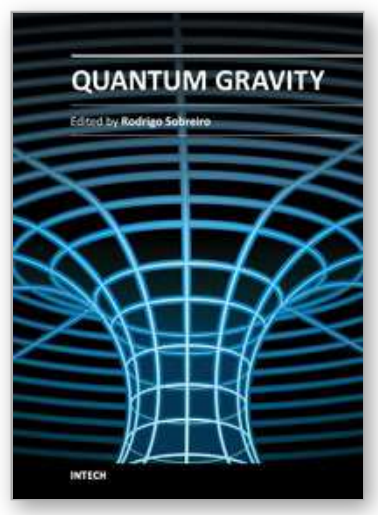

\author{
Quantum Gravity \\ Edited by Dr. Rodrigo Sobreiro
}

ISBN 978-953-51-0089-8

Hard cover, 100 pages

Publisher InTech

Published online 15, February, 2012

Published in print edition February, 2012

The unification between gravity and quantum field theory is one of the major problems in contemporary fundamental Physics. It exists for almost one century, but a final answer is yet to be found. Although string theory and loop quantum gravity have brought many answers to the quantum gravity problem, they also came with a large set of extra questions. In addition to these last two techniques, many other alternative theories have emerged along the decades. This book presents a series of selected chapters written by renowned authors. Each chapter treats gravity and its quantization through known and alternative techniques, aiming a deeper understanding on the quantum nature of gravity. Quantum Gravity is a book where the reader will find a fine collection of physical and mathematical concepts, an up to date research, about the challenging puzzle of quantum gravity.

\title{
How to reference
}

In order to correctly reference this scholarly work, feel free to copy and paste the following:

B.F.L. Ward (2012). Planck Scale Cosmology and Asymptotic Safety in Resummed Quantum Gravity: An Estimate of $\Lambda$, Quantum Gravity, Dr. Rodrigo Sobreiro (Ed.), ISBN: 978-953-51-0089-8, InTech, Available from: http://www.intechopen.com/books/quantum-gravity/planck-scale-cosmology-and-asymptotic-safety-inresummed-quantum-gravity-an-estimate-of-lambda

\section{INTECH}

open science | open minds

\author{
InTech Europe \\ University Campus STeP Ri \\ Slavka Krautzeka 83/A \\ 51000 Rijeka, Croatia \\ Phone: +385 (51) 770447 \\ Fax: +385 (51) 686166 \\ www.intechopen.com
}

\author{
InTech China \\ Unit 405, Office Block, Hotel Equatorial Shanghai \\ No.65, Yan An Road (West), Shanghai, 200040, China \\ 中国上海市延安西路65号上海国际贵都大饭店办公楼 405 单元 \\ Phone: +86-21-62489820 \\ Fax: $+86-21-62489821$
}


(C) 2012 The Author(s). Licensee IntechOpen. This is an open access article distributed under the terms of the Creative Commons Attribution 3.0 License, which permits unrestricted use, distribution, and reproduction in any medium, provided the original work is properly cited. 